Arq. Bras. Med. Vet. Zootec., v.56, n.6, p.715-722, 2004

\title{
Fechamento parcial do anel inguinal externo em eqüinos: avaliação pós-operatória e influência na morfologia testicular
}

\author{
[Partial closure of the equine external inguinal ring: post-operatory evaluation \\ and influence on testicular morphology] \\ J.J.M. Rio Tinto ${ }^{1}$, G.E.S. Alves ${ }^{2}$, R.L. Santos ${ }^{2}$, R.R. Faleiros ${ }^{2}$, A.P. Marques Júnior ${ }^{2}$, E.G. Melo ${ }^{2}$ \\ ${ }^{1}$ Centro de Estudos em Clínica e Cirurgia de Animais - Pontifícia Universidade Católica \\ Rua do Rosário, 1600 \\ 32630000 - Betim, MG \\ ${ }^{2}$ Escola de Veterinária da UFMG - Belo Horizonte, MG
}

\section{RESUMO}

Avaliou-se a influência do fechamento parcial do anel inguinal externo (AIE) na morfologia testicular e caracterizaram-se as alterações pós-operatórias decorrentes de sua utilização em seis eqüinos machos. Sob anestesia geral, criou-se um acesso à cavidade vaginal que foi seguido de síntese da túnica vaginal parietal e sutura do AIE ipsilateral em aproximadamente $75 \%$ de seu diâmetro (antímero suturado). No antímero contralateral foi realizado procedimento cirúrgico semelhante, mas a sutura do anel inguinal foi removida imediatamente após sua aplicação (antímero controle). No pós-operatório, os animais foram examinados diariamente por quatro semanas. $\mathrm{O}$ trauma cirúrgico acarretou degeneração testicular no período pós-operatório e o acesso à cavidade vaginal predispôs à formação de aderências testiculares. $\mathrm{O}$ grau de degeneração testicular foi equivalente nos dois antímeros, suturado e controle, e nenhum dos testículos apresentou degeneração. O procedimento mostrou ser uma alternativa viável para o tratamento de hérnias inguinais quando se deseja evitar a orquiectomia.

Palavras-chave: eqüino, testículo, hérnia inguinal, herniorrafia

\begin{abstract}
In order to evaluate the influence of parcial closure of the external inguinal ring on testicular morphology and to characterize the local post-operatory changes, six adult horses were submitted to surgery under general anesthesia. The vaginal cavity was opened and approximately $75 \%$ of the external inguinal ring was sutured (sutured side). The same procedure was performed on the contralateral side, and the sutures of the external inguinal ring were immediately removed (sham side). The horses were examined daily for four weeks. The surgical procedure induced testicular degeneration and adhesion of the testis to the parietal vaginal tunic. Similar levels of testicular degeneration were detected in both sides (sutured and sham), and none of the testes evaluated had severe degeneration. This procedure can be used for the treatment of inguinal herniation as an alternative to castration and complete closure of the external inguinal ring.
\end{abstract}

Keywords: equine, testis, inguinal herniation, herniorraphy

Recebido para publicação em 16 de junho de 2003

Recebido para publicação, após modificações, em 31 de maio de 2004

E-mail: jorgeriotinto@hotmail.com 


\section{INTRODUÇÃO}

As hérnias inguinais são de grande relevância na espécie eqüina devido à sua ocorrência relativamente alta e à elevada taxa de morte observada nos casos de hérnias adquiridas (Van der Velden, 1988b).

Várias modalidades de tratamento têm sido recomendadas. Nos casos de hérnias inguinais, nas quais a redução manual é possível por manipulação externa da bolsa escrotal ou por tração dos órgãos herniados pela via trans-retal, o tratamento conservativo pode ser empregado. Contudo, essas manobras não são isentas de riscos (Schneider et al., 1982; Varner et al., 1991) e não são recomendadas por alguns autores (Weaver, 1987; Stashak, 1993). O tratamento cirúrgico é o mais indicado para a correção das hérnias inguinais adquiridas, estranguladas ou não, sendo também recomendado nos casos de hérnias congênitas, quando não ocorre resolução espontânea até os seis meses de idade ou quando há sinais de encarceramento ou desconforto abdominal (Adams, 1990; Tulleners, 1991). Várias técnicas para o tratamento cirúrgico das hérnias inguinais têm sido descritas e consistem basicamente na herniorrafia. Após acesso à cavidade da túnica vaginal e redução dos órgãos herniados, realizase a ligadura e a amputação do cordão espermático, da túnica vaginal parietal e do músculo cremáster seguidas ou não de fechamento total do anel inguinal externo. Quando a viabilidade do órgão herniado for questionável recomenda-se a sua remoção pelo acesso inguinal ou mediante laparotomia (Kerjes, 1985; Van der Velden, 1988b; Perkins e Frazer, 1994).

Nos casos de hérnias inguinais encarceradas, a víscera herniada produz compressão sobre o cordão espermático e o testículo, resultando em isquemia que usualmente resulta em necrose testicular e necessidade de orquiectomia. Quando não se verifica o encarceramento, o testículo freqüentemente é viável. Isso também pode ocorrer nos encarceramentos em que o tratamento cirúrgico é realizado prontamente. Nessas situações, alguns autores têm utilizado uma modalidade de tratamento cirúrgico que consiste na redução do conteúdo herniário ou sua remoção, quando necessária, seguida do fechamento parcial do anel inguinal externo, permitindo a passagem apenas do cordão espermático. Esse método de tratamento permite evitar a orquiectomia e é utilizado quando se deseja preservar a função reprodutiva (Heinze et al., 1972; Adams, 1990; Alves et al., 2000; Gaughan, 1998).

Schneider et al. (1982) e Varner e Schumacher (1991) consideram que a utilização de técnicas de herniorrafia que priorizem a preservação dos testículos deve ser desencorajada, uma vez que pode resultar em complicações como edema, fibrose e hipoplasia testicular. Para que a espermatogênese ocorra normalmente é necessário que a temperatura testicular seja mantida em torno de $4^{\circ} \mathrm{C}$ abaixo da temperatura corporal, o que é obtida graças ao escroto, à túnica dartos, ao músculo cremáster e ao plexo pampiniforme (Hafez, 1988). Considerando-se que o trauma cirúrgico produz inflamação e aumento da temperatura local, o que reconhecidamente induz à degeneração testicular (McEntee, 1990; Friedman et al., 1991), potencialmente as alterações locais decorrentes da cirurgia poderiam por si produzir degeneração testicular em intensidade suficiente para inviabilizar o tratamento cirúrgico. Além disso, deve ser considerada a possibilidade de que a redução de diâmetro do anel inguinal externo ou o edema nos tecidos vizinhos poderiam produzir compressão do cordão espermático, resultando em isquemia e degeneração testiculares.

O objetivo deste estudo foi determinar a influência do fechamento parcial do AIE e do trauma resultante de procedimentos cirúrgicos realizados na região inguinal sobre a morfologia dos testículos e caracterizar as alterações clínicocirúrgicas ocorrentes no período pós-operatório.

\section{MATERIAL E MÉTODOS}

O estudo utilizou seis eqüinos sexualmente maduros, sem raça definida e de pesos variáveis (Tab. 1). No mínimo de sete dias antes de serem operados, foram everminados com ivermectina na dose de $0,2 \mathrm{mg} / \mathrm{kg}$ e permaneceram em piquetes coletivos. Todos os animais foram considerados saudáveis ao exame clínico e hematológico e apresentaram testículos de consistência normal e ausência de alterações da genitália externa. 
Tabela 1. Características dos eqüinos utilizados e distribuição pelos tratamentos

\begin{tabular}{lcccc}
\hline \multirow{2}{*}{ Eqüino } & $\begin{array}{c}\text { Idade } \\
\text { (anos) }\end{array}$ & $\begin{array}{c}\text { Peso } \\
(\mathrm{kg})\end{array}$ & \multicolumn{2}{c}{ Antímero } \\
\cline { 5 - 6 } & 4,5 & 283 & Suturado & Controle \\
\hline 1 & 5,0 & 261 & Direito & Direito \\
2 & 7,0 & 408 & Esquerdo \\
3 & 4,0 & 267 & Direito & Esqueito \\
4 & 4,0 & 354 & Esquerdo & Direito \\
5 & 5,5 & 266 & Direito & Esquerdo \\
6 & $5,0 \pm 1,1$ & $306+61$ & & \\
Média & & & & \\
\hline
\end{tabular}

Após jejum alimentar de 18 horas, sob anestesia geral inalatória e em decúbito dorsal, o anel inguinal externo foi palpado em um dos antímeros escolhido aleatoriamente. Realizou-se uma incisão de $10 \mathrm{~cm}$ de extensão na pele e no tecido subcutâneo a $2 \mathrm{~cm}$ medialmente ao AIE (antímero suturado). O testículo foi deslocado para a ferida cirúrgica por manipulação externa. A cavidade da túnica vaginal foi acessada por incisão na túnica dartos, fáscia espermática e túnica vaginal parietal e o testículo foi exteriorizado e examinado. A seguir procedeu-se à síntese da túnica vaginal parietal e da fáscia espermática utilizando fio de categute cromado $\mathrm{n}^{\mathrm{o}} 0$ em padrão de Reverdin contínuo. O AIE foi dissecado dos tecidos adjacentes e medido. A partir de sua extremidade cranial foram realizados pontos em " $X$ ", utilizando fio de náilon de calibre $0,70 \mathrm{~mm}$, resultando no fechamento de aproximadamente $75 \%$ de seu diâmetro. A adequação da abertura remanescente do AIE para a passagem do cordão espermático foi avaliada por palpação, considerando como adequada quando fosse possível a introdução da extremidade do dedo indicador junto ao cordão espermático sem provocar pressão excessiva. $\mathrm{O}$ fluxo sangüíneo no cordão espermático após a sutura do AIE foi confirmado, utilizando-se a dopplermetria. Os tecidos sobrejacentes ao AIE e o subcutâneo foram suturados em conjunto com pontos simples separados, utilizando fio categute cromado $\mathrm{n}^{\mathrm{o}}$ 0. A pele foi suturada com pontos simples separados utilizando fio de algodão $n^{\circ} 1$. Em seguida, efetuou-se procedimento semelhante no antímero oposto (antímero controle), mas as suturas foram removidas logo após a realização dos pontos no anel inguinal, de modo que o trauma cirúrgico fosse semelhante em ambos os antímeros e que em cada eqüino apenas um dos anéis inguinais externos permanecesse suturado (Tab. 1).
Após a recuperação da anestesia, os animais foram mantidos em baias individuais por duas semanas e a seguir colocados em piquetes coletivos por mais duas semanas. No pósoperatório, os eqüinos foram medicados, uma vez ao dia a partir do término da cirurgia, com enrofloxacina $(2,5 \mathrm{mg} / \mathrm{kg} \mathrm{IV}$, durante três dias), flunixin meglumine $(1,1 \mathrm{mg} / \mathrm{kg} \mathrm{IV}$, durante cinco dias) e DMSO (50ml de uma solução a $20 \%$ IV, durante cinco dias).

Durante o pós-operatório de quatro semanas, os eqüinos foram examinados diariamente. Os antímeros suturados foram avaliados quanto à presença e intensidade de claudicação do membro pélvico, de dor à palpação da ferida cirúrgica e de edema no escroto, prepúcio, membro pélvico e região ventral do abdome. A intensidade dessas alterações foi quantificada por meio de escores variando de 0 a 9 . O escore 0 correspondeu à ausência de alterações e o 9 , às alterações mais graves. Adicionalmente, foi realizada a mensuração da largura do prepúcio e do diâmetro escrotal, com compasso e paquímetro, como estimativa quantitativa de edema. Os resultados foram correlacionados com aqueles obtidos por meio dos escores. As feridas cirúrgicas foram avaliadas quanto à presença de exsudação, seroma, hematoma, enfisema subcutâneo e deiscência de pontos.

Quatro semanas após a cirurgia, os eqüinos foram sacrificados e necropsiados. À necrópsia, avaliaram-se a consistência testicular e a ocorrência de aderências entre os folhetos visceral e parietal da túnica vaginal, em ambos os antímeros. Amostras de ambos os testículos foram colhidas e fixadas em solução de Bouin, processadas pela técnica rotineira de inclusão em parafina e coradas pela técnica da hematoxilina e eosina (Luna, 1968). Nos cortes histológicos o grau de degeneração testicular foi quantificado por meio de escores, variando de 0 a 4 (Tab. 2), e pela mensuração do diâmetro dos túbulos seminíferos e da altura do epitélio seminífero.

Os resultados obtidos para altura do epitélio seminífero e diâmetro dos túbulos seminíferos nos dois antímeros, controle e suturado, foram submetidos a análise de variância e as médias foram comparadas pelo teste $\mathrm{t}$ de Student. A comparação dos escores de degeneração testicular entre os testículos dos antímeros controle e suturado foi realizada pelo teste de Mann-Whitney (Sampaio, 1998). 
Tabela 2. Caracterização dos escores para degeneração testicular de eqüinos

\begin{tabular}{ll}
\hline Escore & \multicolumn{1}{c}{ Alteração } \\
\hline 0 - Normal & Ausente \\
1 - Discreta & $\begin{array}{l}\text { Presença de células germinativas degeneradas; desprendimento de células germinativas para o } \\
\text { lúmen tubular com formação ou não de células multinucleadas. Presença de vacúolos no epitélio } \\
\text { seminífero. }\end{array}$ \\
2- Moderada & $\begin{array}{l}\text { Acentuada perda de células germinativas, apresentando as remanescentes degeneradas. } \\
\text { Vacuolização acentuada do epitélio seminífero e diminuição da altura do epitélio. }\end{array}$ \\
3 - Acentuada & $\begin{array}{l}\text { Apenas algumas células germinativas picnóticas permanecem no epitélio. Epitélio germinativo } \\
\text { composto quase exclusivamente por células de Sertoli. Espessamento da membrana basal. }\end{array}$ \\
4 - Muito acentuada & $\begin{array}{l}\text { Perda completa do epitélio germinativo, inclusive das células de Sertoli, com substituição por } \\
\text { tecido conjuntivo fibroso. }\end{array}$ \\
\hline
\end{tabular}

\section{RESULTADOS E DISCUSSÃO}

Em nenhum dos eqüinos verificou-se deiscência das suturas de pele e essas foram removidas dez dias após a cirurgia. Nos eqüinos 2 e 4, constatou-se a presença de pequena quantidade de secreção purulenta na ferida cirúrgica do antímero suturado após o sexto dia do pósoperatório, que desapareceu depois da remoção das suturas. Neles a ferida cirúrgica ficou localizada na região de encontro entre a face medial da coxa e o abdome, onde a retenção de umidade é facilitada, o que, somado à capilaridade e elevada reatividade do fio de algodão, possivelmente resultou na infecção ao redor das suturas. Provavelmente a utilização de fios menos reativos e isentos de capilaridade, como o fio de náilon monofilamentar, e a realização da incisão de pele medialmente ao AIE permitiriam evitar essa complicação. Nenhum eqüino apresentou hematoma na ferida cirúrgica ou enfisema subcutâneo na região inguinal. Apesar de Van der Velden (1988) recomendar a utilização de drenos de Penrose após o acesso ao anel inguinal externo, essa conduta parece ser desnecessária, o que está de acordo com as recomendações de Livesey (1990), uma vez que em nenhum dos antímeros dos eqüinos foi verificada a formação de seroma na ferida cirúrgica.

Os escores médios para as alterações pósoperatórias decorrentes do fechamento parcial do AIE estão representadas nas Fig. 1 e 2 . O fechamento parcial do AIE não resultou em trauma excessivo, uma vez que as alterações locais foram de pequena intensidade, conforme evidenciado pelos escores, que corresponderam, no máximo, a alterações leves. As alterações associadas à dor (dor local e claudicação) foram mais intensas no início do período pós-operatório e diminuíram progressivamente ao longo do tempo. A claudicação foi evidente apenas na primeira semana do pós-operatório. Após a terceira semana, a palpação da ferida cirúrgica já não produzia dor. As alterações locais associadas ao edema foram mais tardias, possivelmente devido à influência da medicação antiinflamatória empregada nos quatro primeiros dias do pós-operatório.

No eqüino $\mathrm{n}^{\mathrm{o}} 5$ foram verificados sinais de comprometimento vascular do testículo após o término da cirurgia, que compreenderam aumento de volume local acompanhado de aumento da sensibilidade, possivelmente decorrentes do fechamento excessivo do AIE, resultando em compressão do cordão espermático. A orquite proveniente de isquemia tem sido considerada como uma das complicações do fechamento parcial do AIE (Livesey, 1990; Perkins e Frazer, 1994). Para evitá-la é necessária uma avaliação cuidadosa da adequação do remanescente do anel inguinal suturado à passagem do cordão espermático. Ao contrário do esperado, o testículo comprometido não apresentou degeneração acentuada e teoricamente o grau de lesão produzida seria passível de regressão.

A correlação entre a largura do prepúcio e a intensidade do edema no prepúcio estimado por escores foi de $98,2 \%$, e a correlação entre o diâmetro escrotal e o escore para o edema da bolsa escrotal foi de $98,3 \%$, o que atesta a eficiência dos escores utilizados para quantificar as alterações pós- operatórias. 

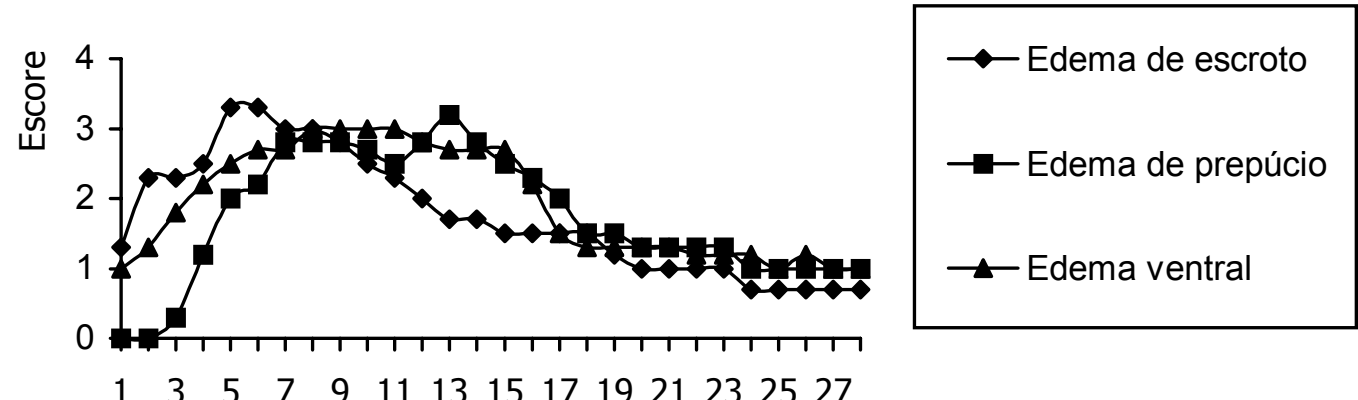

$\begin{array}{llllllllllllll}1 & 3 & 5 & 7 & 9 & 11 & 13 & 15 & 17 & 19 & 21 & 23 & 25 & 27\end{array}$

Dias

Figura 1. Escores médios para edema de escroto, de prepúcio e edema ventral no antímero suturado de eqüinos.
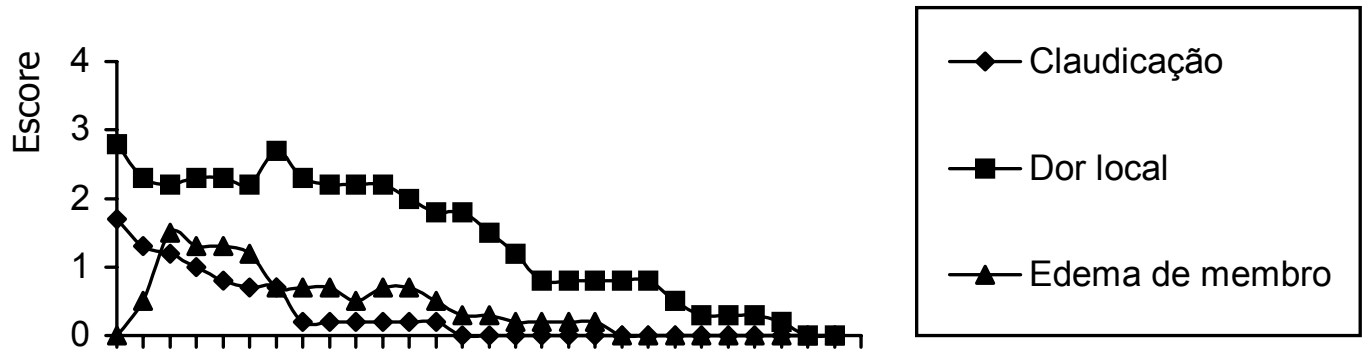

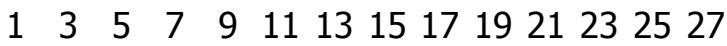

Dias

Figura 2. Escores médios para claudicação do membro pélvico, dor local e edema do membro no antímero suturado de eqüinos.

À necrópsia verificou-se que em ambos os antímeros todos os testículos apresentavam consistência flácida e aderências entre as túnicas vaginais visceral e parietal. De acordo com Roberts (1971), a redução na consistência do testículo é um achado comum nos casos de degeneração testicular e decorre da redução do epitélio seminífero. Provavelmente a ocorrência de aderências testiculares está relacionada ao trauma decorrente da abertura da cavidade vaginal.

Verificou-se a ocorrência de degeneração em todas as amostras de testículos (Tab. 3 e Fig. 3).
O testículo é extremamente sensível a aumentos de temperatura e de acordo com Friedman et al. (1991), períodos de insulação testicular de apenas $48 \mathrm{~h}$ produzem degeneração testicular. Desse modo, provavelmente a degeneração testicular resultou da interferência no processo de termorregulação testicular devido ao aumento de temperatura local devido ao processo inflamatório causado pelo trauma cirúrgico. Apesar de a média dos escores para degeneração testicular nos antímeros suturados ter sido maior do que a dos antímeros controle, essa diferença não foi significativa $(\mathrm{P}=0,13)$, indicando que $\mathrm{o}$ fechamento parcial do AIE não agravou a 
degeneração testicular decorrente do trauma cirúrgico. A estimativa da degeneração testicular pela mensuração do diâmetro dos túbulos seminíferos e da altura do epitélio seminífero (Tab. 4) também não revelou diferenças significativas entre os antímeros controle e suturado (respectivamente $\mathrm{P}=0,71$ e $\mathrm{P}=0,47$ ), confirmando os resultados citados anteriormente, e indica que o trauma cirúrgico constitui a principal fonte de lesão para o testículo.
Tabela 3. Escores para degeneração testicular nos testículos dos antímeros controle e suturado de eqüinos

\begin{tabular}{lcc}
\hline Eqüino & \multicolumn{2}{c}{ Antímero } \\
\cline { 2 - 3 } & Suturado & Controle \\
\hline 1 & 1,25 & 1,75 \\
2 & 2,75 & 2,00 \\
3 & 2,25 & 1,25 \\
4 & 2,75 & 2,50 \\
5 & 2,75 & 1,50 \\
6 & 1,75 & 1,00 \\
Média & $2,25 \mathrm{a}$ & $1,67 \mathrm{a}$ \\
\hline \multicolumn{2}{l}{ Médias seguidas de letras iguais são equivalentes $(\mathrm{P}>0,05)}$.
\end{tabular}

Tabela 4. Diâmetro dos túbulos seminíferos e altura do epitélio seminífero nos testículos dos antímeros controle e suturado de eqüinos

\begin{tabular}{lccccc}
\hline \multirow{2}{*}{ Eqüino } & \multicolumn{2}{c}{ Diâmetro dos túbulos seminíferos } & & \multicolumn{2}{c}{ Altura do epitélio seminífero } \\
\cline { 2 - 3 } \cline { 5 - 6 } & Antímero suturado & Antímero controle & & Antímero suturado & Antímero controle \\
\hline 1 & 319,9 & 251,9 & 209,5 & 84,5 & 71,4 \\
2 & 227,0 & 261,8 & & 39,7 & 48,3 \\
3 & 284,4 & 249,1 & 56,9 & 61,2 \\
4 & 243,6 & 241,6 & & 40,1 & 42,7 \\
5 & 222,5 & 263,9 & & 45,5 & 68,2 \\
6 & 222,5 & $246,3 \pm 19,8 \mathrm{a}$ & & $54,1 \pm 16,9 \mathrm{a}$ & $60,6 \pm 12,4 \mathrm{a}$ \\
\hline
\end{tabular}

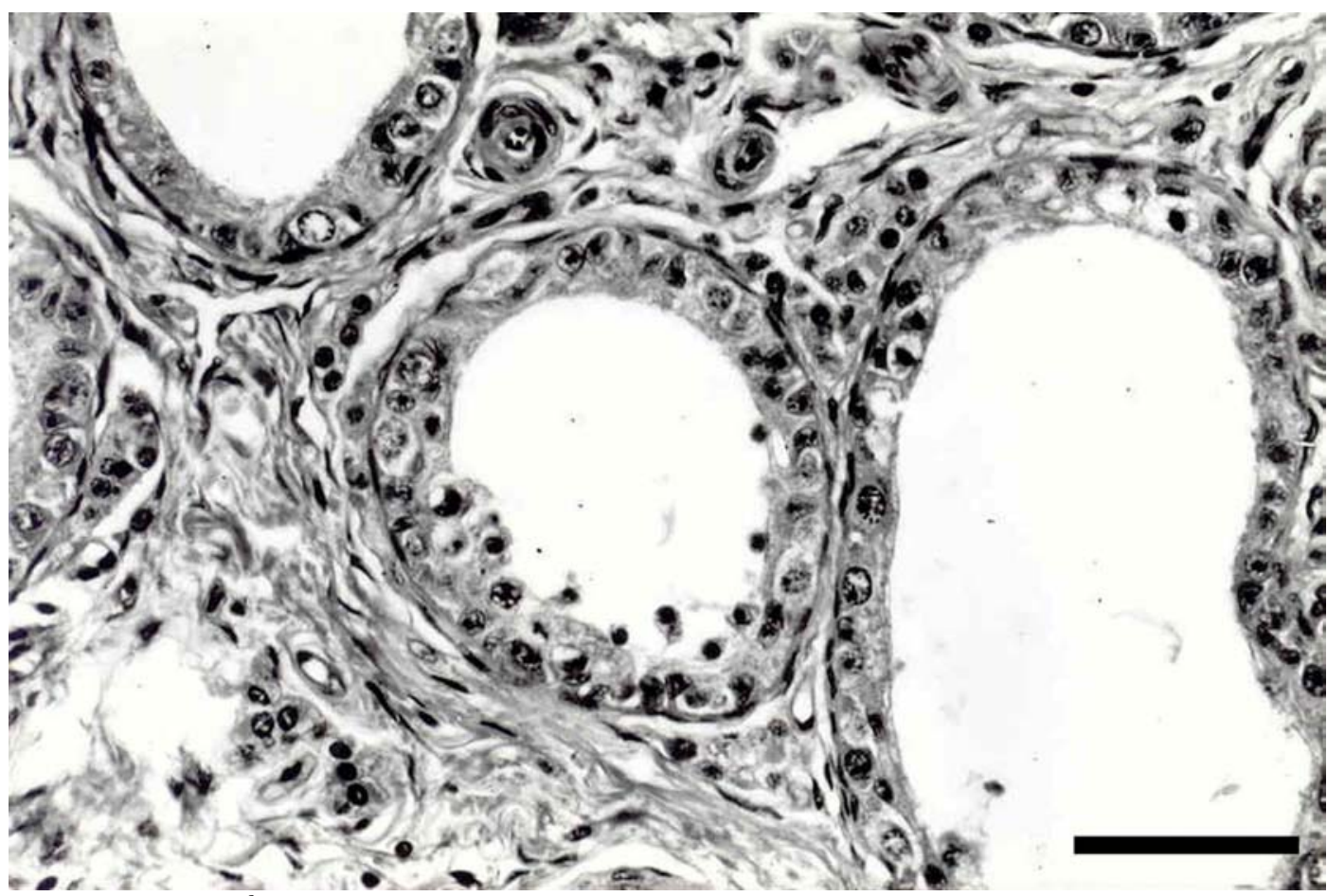

Figura 3. Eqüino $\mathrm{n}^{\mathrm{o}}$ 5. Degeneração testicular moderada no antímero suturado, correspondente ao escore 2,75. Hematoxilina e eosina, barra $=50 \mu \mathrm{m}$. 
Segundo Roberts (1971), o grau de degeneração depende do tempo de exposição dos testículos às condições adversas, sendo que esta pode ser irreversível quando ocorre destruição das camadas basais do epitélio germinativo, incluindo as espermatogônias e as células de Sertoli. Como não se verificou degeneração acentuada em nenhum dos testículos examinados e considerando que, segundo Friedman et al. (1991), a recuperação da degeneração testicular é um processo lento, pode-se dizer que a tendência das alterações histopatológicas observadas seria de regressão com o passar do tempo, uma vez que $o$ processo inflamatório reduziu-se progressivamente durante o pós-operatório. A longo prazo, as aderências testiculares verificadas poderiam interferir no processo de termorregulação, comprometendo a recuperação das alterações existentes.

Várias modalidades de tratamento cirúrgico têm sido empregadas para as hérnias inguinais nos eqüinos, com ou sem a orquiectomia. A etiopatogenia das hérnias inguinais em eqüinos é controversa, assim como a indicação de tratamentos cirúrgicos que permitam evitar a orquiectomia.

Alguns autores, baseados na hipótese de que essa enfermidade possui origem hereditária, recomendaram a orquiectomia bilateral em todos os casos, sejam da forma congênita ou adquirida (Walker e Vaughan, 1980; Mcllwraight e Turner, 1987; Livesey, 1990; Stashak, 1993). Entretanto, como não se encontram disponíveis até o momento estudos que comprovem a hereditariedade das hérnias adquiridas, outros autores indicaram apenas a remoção do testículo ipsilateral à hérnia (Schneider, 1982; Kerjes et al., 1985; Weaver, 1987; Van der Velden, 1988). Heinze et al. (1972), Tulleners, (1990) e Robertson (1991) não fizeram restrições à tentativa de preservação do testículo e descreveram técnicas que podem ser empregadas com esse objetivo.

Os resultados permitem concluir que a realização de cirurgias na região inguinal acarreta degeneração testicular. $\mathrm{O}$ processo degenerativo não é intensificado pelo fechamento de aproximadamente $75 \%$ do anel inguinal externo e, portanto, esse procedimento constitui uma alternativa viável para o tratamento de hérnias inguinais quando se deseja evitar a orquiectomia

\section{REFERÊNCIAS BIBLIOGRÁFICAS}

ADAMS, R. The urogenital system. In: KOTERBA, A.M.; DRUMMOND, W.H.; KOSCH, P.C. (Eds.). Equine clinical neonatology. Malvern: Lea \& Febiger, 1990. P.443-495.

ALVES, G.E.S.; SANTOS, R.L.; HENRY, M.R.J.M. et al. Acquired bilateral inguinal hernia in a stallion. Equine Vet. Educ., v.12, p.256-259, 2000 .

FRIEDMAN, R.; BULLARD, J.F.; JOHNSON, L.E. The effects of increased testicular temperature on spermatogenesis in the stallion. $J$. Reprod. Fertil., v.44, p.127-134, 1991.

GAUGHAN, E.M. Inguinal hernias in horses. Comp. Cont. Educ. Pract. Vet., v.20, p.10571059. 1998.

HAFEZ, E.S.E. Reprodução animal. 4.ed. São Paulo: Manole, 1998. 720p.

HEINZE, C.D.; BULLARD, J.F.; JOHNSON, L.E. Hernias. In: CATCOTT, E.J.; SMITHCORS, J.F. (Eds.). Equine medicine and surgery. 2.ed. Wheaton: American Veterinary Publications, 1972. P.852-862.

KERJES, A.W.; NEMETH, F.; RUTGERS, J.E. A colour atlas of large animal surgery. Utretch: Wolfe Medical Publications, 1985. P.51-75.

LIVESEY, M.A. Inguinal hernia. In: WHITE, N.A.; MOORE, J.N. (Eds.). Current practice in equine surgery. Philadelphia: Lea \& Febiger, 1990. P.321-327.

LUNA, L.G. Manual of histologic staining methods of the Aimed Forces Institute of Pathology. 3.ed. New York: McGraw Hill, 1968. 257p.

McENTEE, K. Reproductive pathology of domestic mammals. Academic, SNC, 1990. $401 \mathrm{p}$.

MCILWRAIGHT, C.W.; TURNER, A.S. Equine surgery advanced techniches. Philadelphia: Lea \& Febiger, 1987. P.333-338.

PERKINS, N.P.; FRAZER, G. Reproductive emergencies in the stallion. Vet. Clin. North Am.: Equine Pract., v. 10, p. 671-683, 1991.

ROBERTS, S.J. Veterinary obstetrics and genital diseases (Theriogenology). Woodstock: Roberts, 1971. 776p. 
ROBERTSON, J.T. Diseases of the alimentary system In: COLAHAN, P.T.; MAYHEW, I.G.; MERRIT, A.M. et al. (Eds.). Equine medicine and surgery. 4.ed. Goleta: American Veterinary Publications, 1991. P.683-704.

SCHNEIDER, R.K.; MILNE, D.W.; KOHN, C.W. Acquired inguinal hernia in the horse: a review of 27 cases. J. Am. Vet. Med. Assoc., v.180, p.317-320, 1982.

STASHAK, T.S. Inguinal hernia. In: McKINNON, A.O.; VOSS, J.L. (Eds.). Equine reproduction. Malvern: Lea \& Febiger, 1993. P.925-932.

TULLENERS, E.P. Diseases of the alimentary system In: COLAHAN, P.T.; MAYHEW, I.G.; MERRIT, A.M. et al. (Eds.). Equine medicine and surgery. 4.ed. Goleta: American Veterinary Publications, 1991. P.609-616.
VAN DER VELDEN, M.A. Ruptured inguinal hernia in new-born colt foals: a review of 14 cases. Equine Vet. J., v.20, p.178-181, 1988.

VARNER, D.D.; SCHUMACHER, J. Diseases of the reproductive system. In: COLAHAN, P.T.; MAYHEW, I.G.; MERRIT, A.M. et al. (Eds.). Equine medicine and surgery. 4.ed. Goleta: American Veterinary Publications, 1991. P.911914.

WALKER, D.F.; VAUGHAN, J.T. Bovine and equine urogenital surgery. Philadelphia: Lea \& Febiger, 1980. P.163-165.

WEAVER, A.D. Acquired incarcerated inguinal hernia: a review of 13 horses. Can. Vet. J., v.28, p.195-199, 1987. 